\title{
Chronic liver disease independently associated with COVID-19 severity: evidence based on adjusted effect estimates
}

\author{
Haiyan Yang ${ }^{1} \mathbb{D} \cdot \mathrm{Jie}_{\mathrm{Xu}}{ }^{1} \cdot$ Xuan Liang $^{1} \cdot \mathrm{Li} \mathrm{Shi}^{1} \cdot$ Yadong Wang $^{2}$ \\ Received: 15 December 2020 / Accepted: 31 December 2020 / Published online: 28 January 2021 \\ (c) Asian Pacific Association for the Study of the Liver 2021
}

Keywords COVID-19 $\cdot$ Chronic liver disease $\cdot$ Severity $\cdot$ Adjusted effect estimate $\cdot$ Meta-analysis

Recently, a meta-analysis by Kovalic et al. reported that chronic liver disease was significantly associated with severe coronavirus disease 2019 (COVID-19) and mortality [1]. This is an interesting study. However, the pooled effect on the association between chronic liver disease and severe COVID-19 was estimated based on un-adjusted effect sizes in Kovalic et al.'s study [1]. It has been considered that several factors such as gender, age and certain comorbidities significantly influenced COVID-19 outcomes [2-5]. This suggests that these factors might modulate the relationship between chronic liver disease and COVID-19 severity. Therefore, it is urgently required to clarify this association by performing a quantitative meta-analysis based on adjusted effect estimates.

Electronic databases including PubMed, Web of Science and EMBASE were searched up to December 10, 2020 using the terms: "SARS-CoV-2", "COVID-19", "chronic liver disease", "cirrhosis", "hepatitis", "liver cancer" and "nonalcoholic fatty liver disease". Only studies reporting the relationship between chronic liver disease and COVID19 severity by adjusted effect estimates were included. Case reports, reviews, duplicate publications, errata and studies

\section{Supplementary Information The online version contains supplementary material available at https://doi.org/10.1007/s1207 2-020-10133-y.}

\section{Haiyan Yang \\ yhy@zzu.edu.cn \\ $\triangle$ Yadong Wang wangyd76@163.com}

1 Department of Epidemiology, School of Public Health, Zhengzhou University, No. 100 of Science Avenue, Zhengzhou 450001, China

2 Department of Toxicology, Henan Center for Disease Control and Prevention, No. 105 of South Nongye Road, Zhengzhou 450016, China without sufficient data were excluded. The heterogeneity was detected by $I^{2}$ statistics. The pooled effect sizes with $95 \%$ confidence interval (CI) were estimated. Publication bias was evaluated by Begg's test and Egger's test. Sensitivity analysis, subgroup analysis and meta-regression analysis were also performed. All data were analyzed using Stata 12.1. $p<0.05$ was considered statistical significance.

Figure S1 shows the flow diagram of study selection. 29 articles with 90,095 confirmed COVID-19 patients were included. The characteristics of the included studies are summarized in Table 1. Our meta-analysis based on adjusted effect estimates demonstrated that COVID-19 patients with chronic liver disease tended to develop severe outcome compared to those without (pooled effect size $=1.52,95 \%$ CI: 1.14-2.02, Fig. 1a) and had a significantly increased risk for mortality compared to those without (pooled effect size $=1.36,95 \%$ CI: $1.22-1.53$, Fig. 1b). Sensitivity analysis exhibited that our findings were stable (Fig. 1c). Subgroup analyses by sample size and study design exhibited consistent results (Table S1 and Figure S2-3). But inconsistent results were observed in subgroup analyses by age, male percentage, effect estimate and region (Table S1 and Figure S4-7). Meta-regression analysis showed that the tested variables such as sample size, age, male percentage, effect estimate, study design and region might not be the source of heterogeneity (Table S1). Begg's test and Egger's test suggested that there might be potential publication bias (Figure S8).

This meta-analysis has several limitations. First, inconsistent results were observed in subgroup analyses by age, male percentage and region. Thus, the findings should be cautiously extrapolated to whole population. Second, most of the included studies are retrospective, further welldesigned studies with more prospective literatures are warranted to confirm our findings. Third, publication bias might 


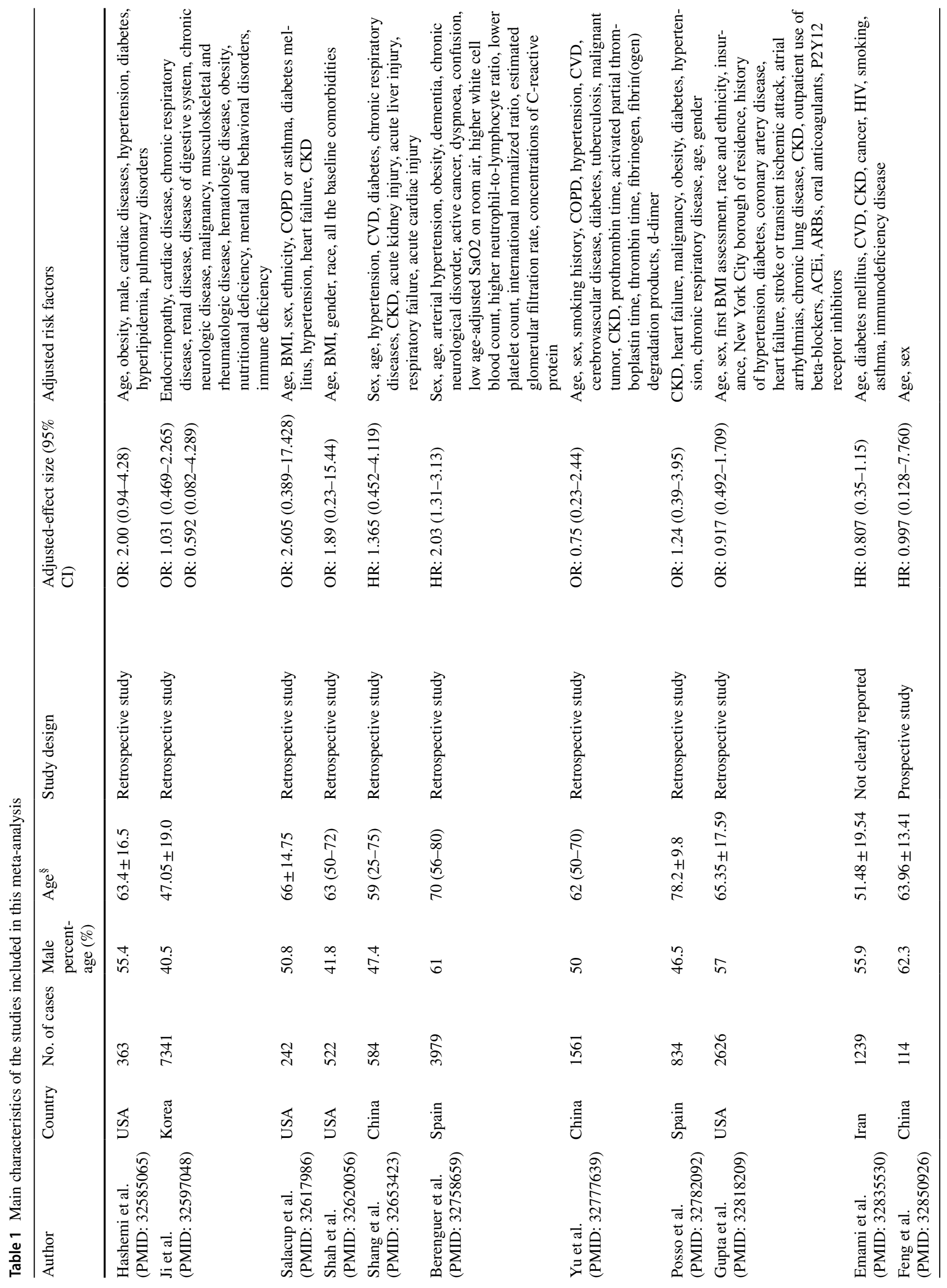




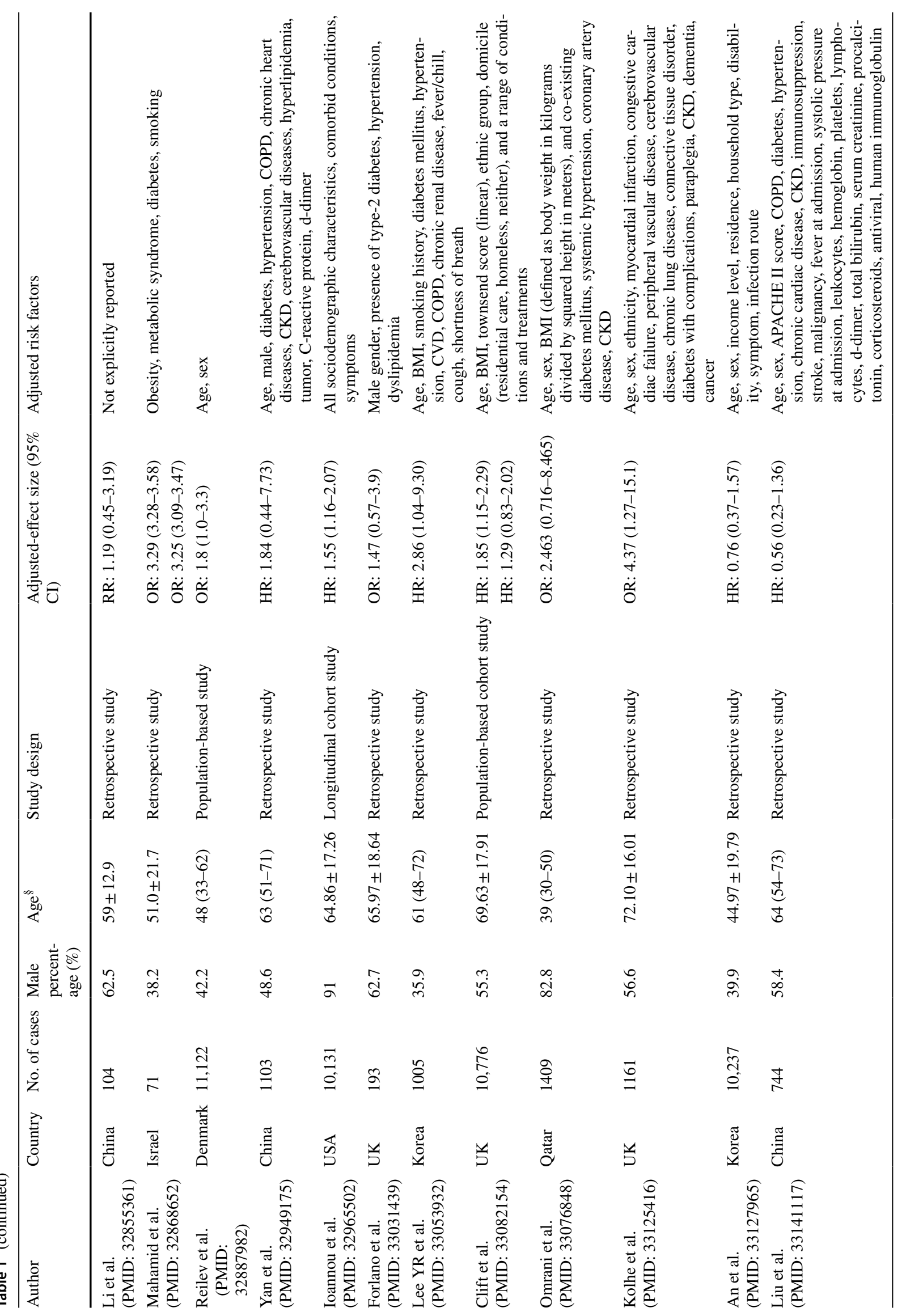




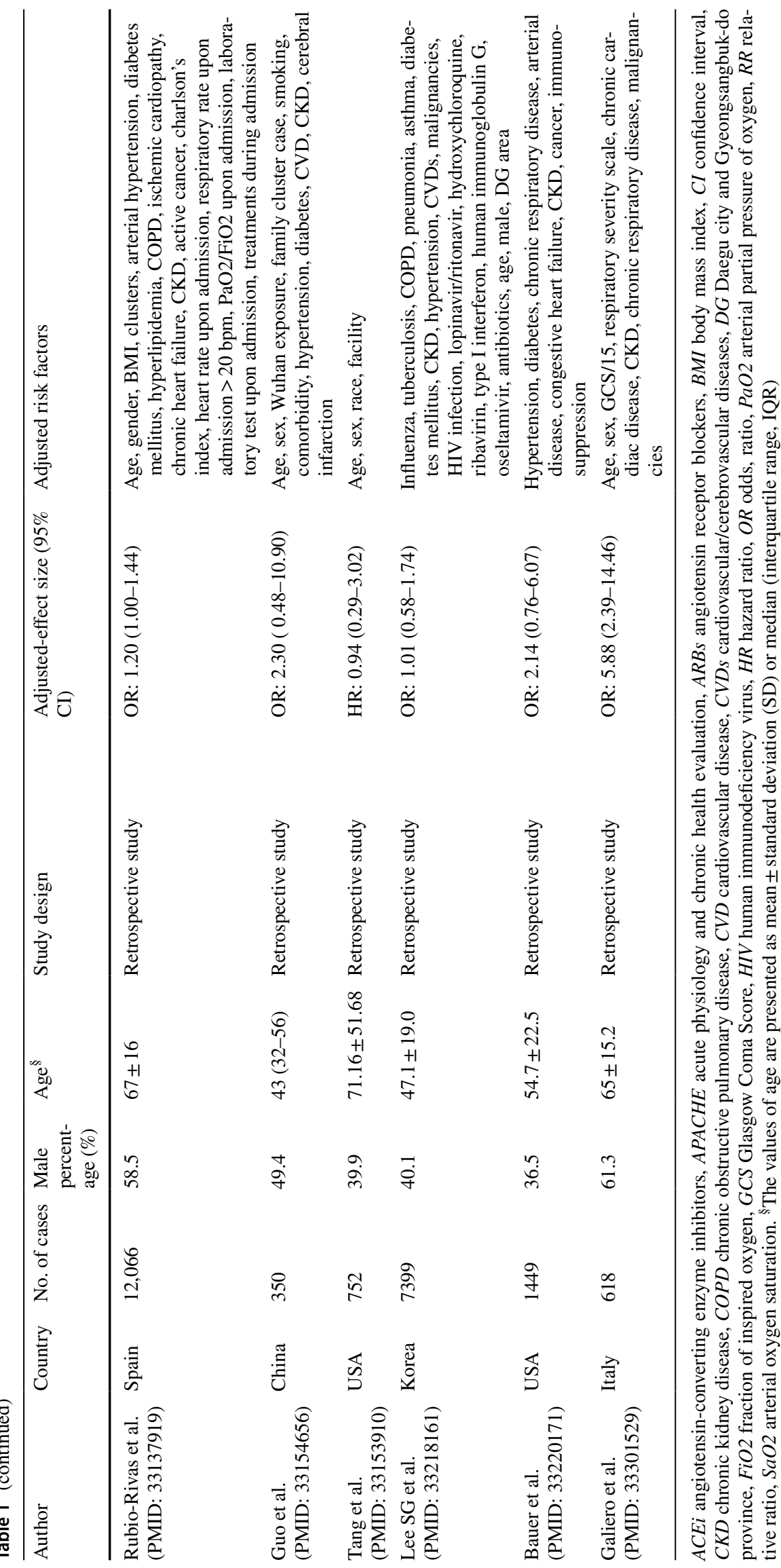


a

\begin{tabular}{ll|lll} 
Study \\
ID
\end{tabular}

b

Study
ID

C

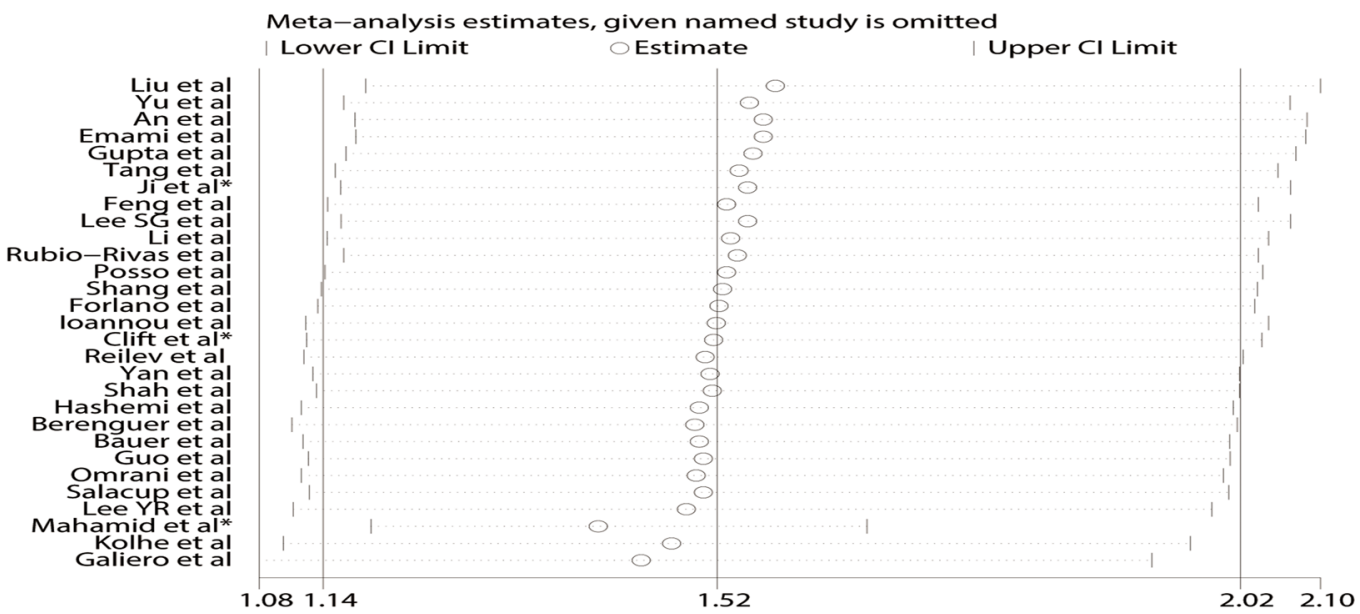

Fig. 1 a The forest plot on the association between chronic liver disease and severe coronavirus disease 2019 (COVID-19) on the basis of 29 studies with 90,095 cases reporting adjusted effect estimates; b The forest plot on the association between chronic liver disease and
COVID-19 mortality; c Leave-one-out sensitivity analysis indicated that our results were stable and robust. *Indicates that the combined value was calculated on the basis of subgroups 
exist although we tried to search potential articles in electronic databases.

In summary, our study indicated that chronic liver disease was independently associated with COVID-19 severity and mortality, especially among aged individuals, male-dominated population, USA and Europe. Proper management of COVID-19 patients with chronic liver disease is highly recommended to prevent severe situations and mortality.

Acknowledgements We would like to thank Ying Wang, Hongjie Hou, Peihua Zhang, Yang Li, Jian Wu and Wenwei Xiao (All are from Department of Epidemiology, School of Public Health, Zhengzhou University, Zhengzhou 450001, China) for their kind help in searching articles and collecting data, and valuable suggestions for data analysis.

Author contributions Haiyan Yang and Yadong Wang designed this study. Jie $\mathrm{Xu}$ and Xuan Liang performed literature search. Haiyan Yang and Jie Xu performed data extraction. Jie Xu, Haiyan Yang, Xuan Liang and Li Shi performed statistical analyses. Haiyan Yang, Jie Xu and Yadong Wang wrote and reviewed the manuscript. All the authors approved the final version of this manuscript.

Funding This study was supported by grants from National Natural Science Foundation of China (Grant Number 81973105), Key Scientific Research Project of Henan Institution of Higher Education (Grant Number 21A330008) and Joint Construction Project of Henan Medical Science and Technology Research Plan (Grant Number LHGJ20190679). The funders have no role in the data collection, data analysis, preparation of manuscript and decision to submission.

Data availability All data relevant to this study are included in this article or uploaded as supplementary information.

\section{Compliance with ethical standards}

Conflict of interest The authors Haiyan Yang, Jie Xu, Xuan Liang, Li Shi and Yadong Wang have no any potential conflict of interest regarding this submitted manuscript.

\section{References}

1. Kovalic AJ, Satapathy SK, Thuluvath PJ. Prevalence of chronic liver disease in patients with COVID-19 and their clinical outcomes: a systematic review and meta-analysis. Hep Intl 2020;14(5):612-20

2. Liang X, Shi L, Wang Y, Xiao W, Duan G, Yang H, et al. The association of hypertension with the severity and mortality of COVID-19 patients: evidence based on adjusted effect estimates. J Infect 2020;81(3):e44-7

3. Biswas M, Rahaman S, Biswas TK, Haque Z, Ibrahim B. Association of sex, age, and comorbidities with mortality in COVID-19 patients: a systematic review and meta-analysis. Intervirology 2020;9:1-12

4. Xu J, Xiao W, Liang X, Zhang P, Shi L, Wang Y, et al. The association of cerebrovascular disease with adverse outcomes in COVID-19 patients: a meta-analysis based on adjusted effect estimates. J Stroke Cerebrovasc Dis 2020;29(11):105283

5. Dorjee K, Kim H, Bonomo E, Dolma R. Prevalence and predictors of death and severe disease in patients hospitalized due to COVID19: a comprehensive systematic review and meta-analysis of 77 studies and 38,000 patients. PLoS One 2020;15(12):e0243191

Publisher's Note Springer Nature remains neutral with regard to jurisdictional claims in published maps and institutional affiliations. 